\title{
CT EVALUATION OF THE EQUIVOCAL PULMONARY NODULE
}

\author{
Murray A. Howe and Barry H. Gross* \\ Department of Radiology, University of Michigan Medical School, Ann Arbor, MI 48109, U.S.A.
}

(Received 11 August 1986; received for publication 6 November 1986)

\begin{abstract}
In the setting of a questionable pulmonary nodule demonstrated by conventional radiographs, the place of CT in the diagnostic algorithm is not well established. We reviewed our experience in 50 consecutive patients referred to CT for a "possible pulmonary nodule." From the chest radiographs we noted nodule location, maximum dimension, presence on one or both views, and presence on a previous radiograph ( $>1$ year old), and nodules were categorized as "likely" or "unlikely" to be real parenchymal lesions based on radiographic appearance. Of a total of 56 questionable nodules, CT demonstrated no abnormality in 21 cases. parenchymal nodules in 16, scarring, atelectasis, or infiltrate in 11 , and normal structural variants in 8 . True pulmonary nodules were statistically significantly more frequently categorized as "likely" lesions than normal variants or no disease, but this was not of a magnitude to be clinically useful. Based on analysis of various radiographic features of equivocal nodules and their subsequent outcomes, we suggest a radiologic approach to the equivocal pulmonary nodule.
\end{abstract}

Lung, CT Lung neoplasms, diagnosis Thorax, radiugraphy Thorax, CT

\section{INTRODUCTION}

Computed tomography (CT) has assumed a major role in the evaluation of pulmonary parenchymal nodules in a variety of clinical settings. In patients with extrathoracic primary malignant neoplasms, CT is often used to detect pulmonary metastases, even when conventional chest radiographs are normal. In patients with a solitary pulmonary nodule demonstrated by conventional radiographs, CT densitometry may be the most effective way to determine the presence of calcification [1]. When a solitary nodule is felt to represent bronchogenic carcinoma, CT is considered by many to be an efficacious tool for noninvasive staging of disease extent [2].

In the clinical setting of a questionable nodule demonstrated by conventional radiographs, the place of $\mathrm{CT}$ in the diagnostic algorithm is not as well established. We reviewed our experience in 50 consecutive patients referred to CT for a "possible pulmonary nodule." The results of this review and our recommendations for this common clinical situation form the basis of this report.

\section{MATERIALS AND METHODS}

We retrieved the CT records at our hospital from 1 January 1982 to 15 August 1985 to identify all patients evaluated for the presence of one or more equivocal pulmonary nodules. We reviewed the corresponding posteroanterior (PA) and lateral chest radiographs without knowledge of the clinical history or the CT findings and noted the following parameters: nodule location, maximum dimension, presence on one or both views, and presence on a previous radiograph (greater than 1 year-old). Nodules were also categorized as "likely" or "unlikely" to be real parenchymal lesions based on their radiographic appearance. We then revicwed the CT scans to look for findings to explain the lesions in question. CT scan findings were categorized as follows: "nodule," "clear-cut scarring, atelectasis, or infiltrate," "normal structural variant," or "no disease noted." If a nodule was present, we looked for calcification or for other nodules. All CT scans were performed on GE 8800 or 9800 CT scanners with scan times of $4.8-5.6 \mathrm{sec}$. Contiguous $1 \mathrm{~cm}$ thick slices were obtained from the lung apices

\footnotetext{
* To whom correspondence and reprint requests should be addressed: Barry H. Gross, M.D., Department of Radiology, BID502/0030, University of Michigan Medical Center, 1500 E. Medical Center Drive, Ann Arbor, MI 48109-0030, U.S.A.
} 
through the lung bases and were photographed at lung (level $=-600 \mathrm{H} . \mathrm{U}$. , width $=1000 \mathrm{H} . \mathrm{U}$.) and soft tissue (level $=30 \mathrm{H} . \mathrm{U}$., width $=500 \mathrm{H}$.U.) windows; repeat contiguous $5 \mathrm{~mm}$ thick slices were sometimes obtained in areas of suspected abnormality.

Careful chart review was then performed addressing the following points: clinical history pertaining to the chest radiograph, subsequent workup, and current patient and lesion status. Clinical indications for chest radiography varied from routine evaluation to symptomatic for possible lung neoplasm (hemoptysis, new onset of cough, weight loss) to known malignancy. Subsequent workup after chest CT included biopsy in four patients. In those patients without pathologic proof of lesion etiology, we attempted careful clinical correlation, including follow-up chest radiography and/or CT, to determine the nature of the pulmonary nodule. For example, nodules responding to antineoplastic therapy synchronously with known malignancy at other sites were considered probably malignant, while nodules that resolved with antibiotic therapy in patients with clinical evidence of infection (fever, leukocytosis) were considered probably inflammatory. Nodules were considered benign if stability or regression was demonstrated for at least 1 year in the absence of antineoplastic therapy.

The statistical workup of these data used paired $\chi^{2}$ analysis.

\section{RESULTS}

Fifty patients with a total of 56 questionable pulmonary nodules at conventional chest radiography underwent CT scanning. Pathologic or clinical confirmation was available in $71 \%$. Overall, 16 of 56 equivocal nodules were shown to be real at CT. Eleven equivocal nodules were explained by scar, atelectasis, or infiltrate (SAI), while 8 represented normal structural variants. CT demonstrated no abnormality for 21 of 56 suspected nodules. Subsequent follow-up of the latter 21 patients revealed no parenchymal neoplasms that were missed by CT. Mean nodule size was similar whether CT showed no disease, a true nodule, SAI, or a normal structural variant (see Table 1). Overall, $61 \%$ of the equivocal nodules in this study were $1 \mathrm{~cm}$ or less in size.

There was no statistical difference in mean patient age or percentage of lesions present on old radiographs for any CT outcome. Similarly, there was considerable overlap in clinical history for all outcomes. True pulmonary nodules were more likely to be seen on two views than no disease (44 vs $15 \%)$, but statistical significance was not achieved $(P=0.12)$.

The assessment of likelihood of a real lesion demonstrated statistically significant differences among the various outcomes. True pulmonary nodules, $81 \%$ of which were categorized as likely lesions, were more often considered real lesions when compared to normal variants $(P=0.0009)$ or no discasc $(P=0.01)$.

In assessing location of the equivocal nodules, we emphasized the relative position of the lesions with respect to ribs and vertebrae. Many equivocal nodules projected over a bony structure (36/56 overall, including 7 of 16 true nodules), but only 1 of 12 lesions that projected over two ribs turned out to be a true nodule (Fig. 1). Furthermore, all four equivocal nodules that projected over the first or second costochondral junction were due to prominence of these junctions (Fig. 2).

Of 16 true nodules, seven were considered benign, including three that were granulomas, one that represented chronic inflammation, one that resolved with antibiotic therapy, one that was diffusely calcified at CT densitometry, and one that was densely calcified and showed no growth over a 2-year period. Of the remaining nodules, four demonstrated interval growth, two were biopsy-proven bronchogenic carcinomas, two were lost to follow-up in patients who died of underlying malignancy, and one responded to antineoplastic therapy. CT revealed multiple nodules in two patients who each had only one lesion seen on conventional chest radiographs.

Of patients with multiple equivocal nodules, one had 1 benign nodule and 2 "no disease noted" in the areas of interest, one had 2 "no disease noted," one had 1 "probably malignant" and 1 "no disease noted," one had 2 biopsy-proven benign nodules, and one had 2 biopsy-proven malignant nodules. Interestingly, seven patients were thought by their referring clinicians to have equivocal pulmonary nodules despite a formal radiologic interpretation of "no pulmonary nodules." CT demonstrated 3 SAI, 2 costochondral junction calcifications, 1 no disease, and 1 true nodule (Fig. 3 ) in this group of patients. The patient with a true nodule had adenocarcinoma of unknown primary metastatic to the liver and died shortly after undergoing chest CT. Autopsy was not performed. 


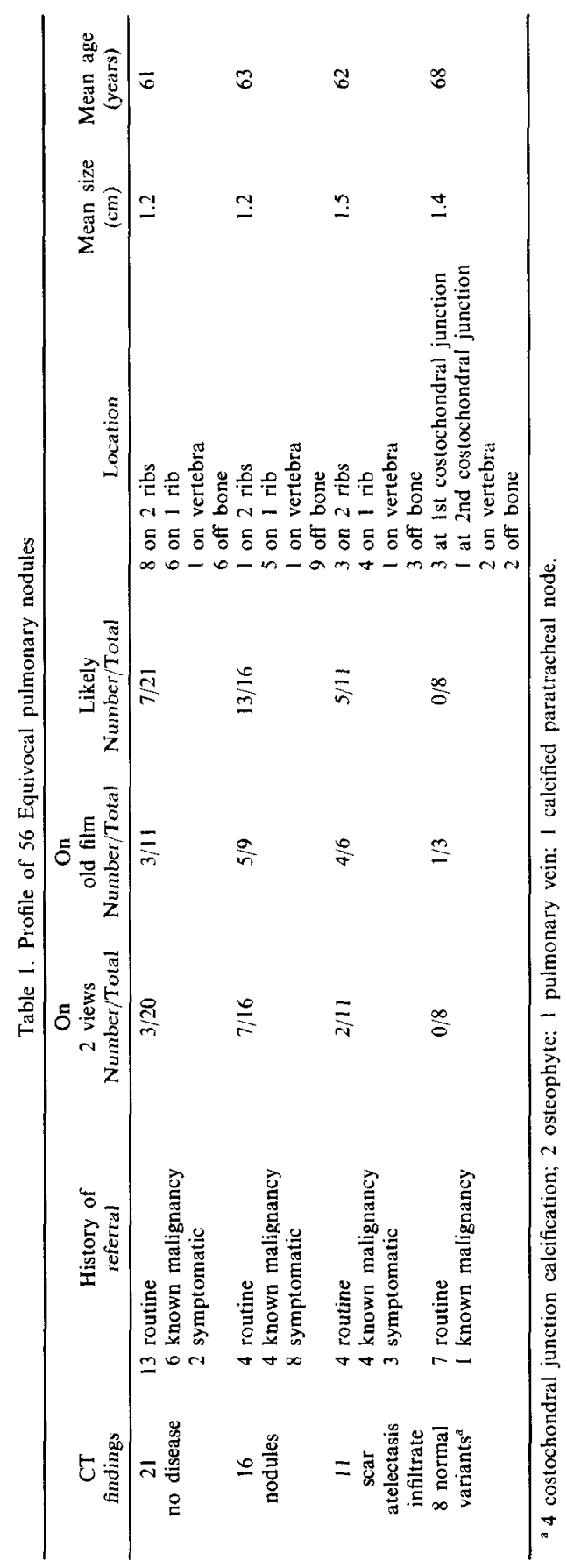




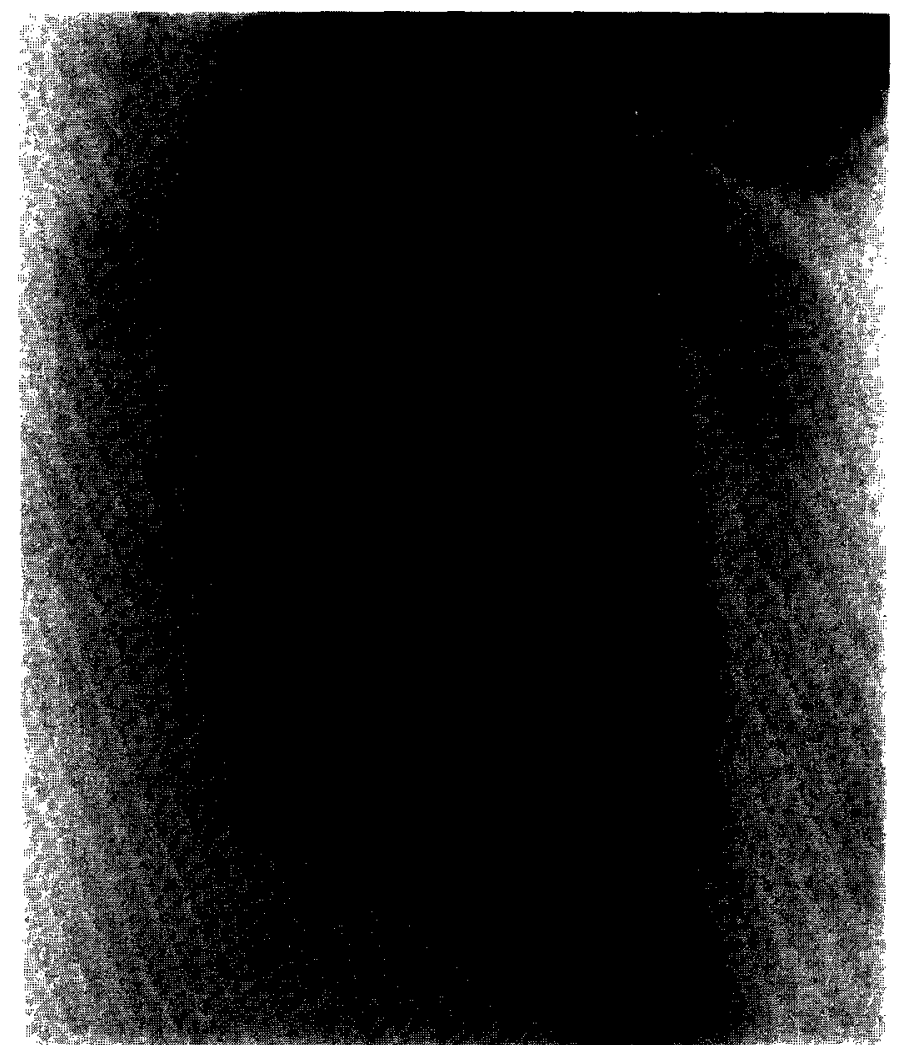

Fig. 1. Equivocal nodule projected over left third anterior and seventh posterior ribs (arrowhead). CT revcaled no abnormality.

\section{DISCUSSION}

The effective management of patients with equivocal pulmonary nodules requires differentiation of true intraparenchymal nodules, normal structural variants, technical artifacts, non-nodular pulmonary abnormalities, and extrapulmonary lesions. While it is of interest that a chest radiologist could characterize equivocal nodules as likely or unlikely to be real lesions with some accuracy, this was clearly not of a magnitude sufficient to serve in the clinical setting. Follow-up conventional chest radiography is another method for establishing the significance of an equivocal nodule, but the diagnostic delay that this entails will not always be clinically acceptable. Other modalities for further evaluation of equivocal nodules have included chest fluoroscopy, oblique chest radiographs, and/or conventional chest tomography. With the advent of CT there has been confusion on the part of clinicians and radiologists as to which studies should be obtained for a questionable nodule.

In our institution the availability of CT has resulted in a marked reduction in the number of conventional tomograms performed, and chest fluoroscopy is virtually never done. The reduced role of conventional tomography is probably well deserved. CT and conventional tomography are roughly similar in terms of cost (approximately $\$ 500$ vs $\$ 300$ in our institution) and radiation dose, and CT has proven to be more sensitive at detecting parenchymal lesions [3-5] and far better at evaluating extraparenchymal abnormalities of the mediastinum, hila, chest wall, and upper abdomen [6].

Oblique chest radiographs are, from the standpoint of the radiologist, perhaps more convenient to order than chest fluoroscopy. However, this convenience is negated in our experience by the frequent nondiagnostic nature of the resultant study. At chest fluoroscopy, it is easier to define the angle of obliquity and degree of inspiration at which an equivocal nodule is viewed to maximum diagnostic advantage.

Chest fluoroscopy has several attractive features in comparison with CT and conventional tomography. It is far less expensive (less than $\$ 100$ in our institution), much faster, more convenient to schedule and perform, and lower in patient radiation dose. The fact that fluoroscopy can often be performed immediately after an equivocal nodule is detected on chest radiographs means that the patient will sometimes be spared the anxiety of waiting for a CT scan to rule out cancer. The major 

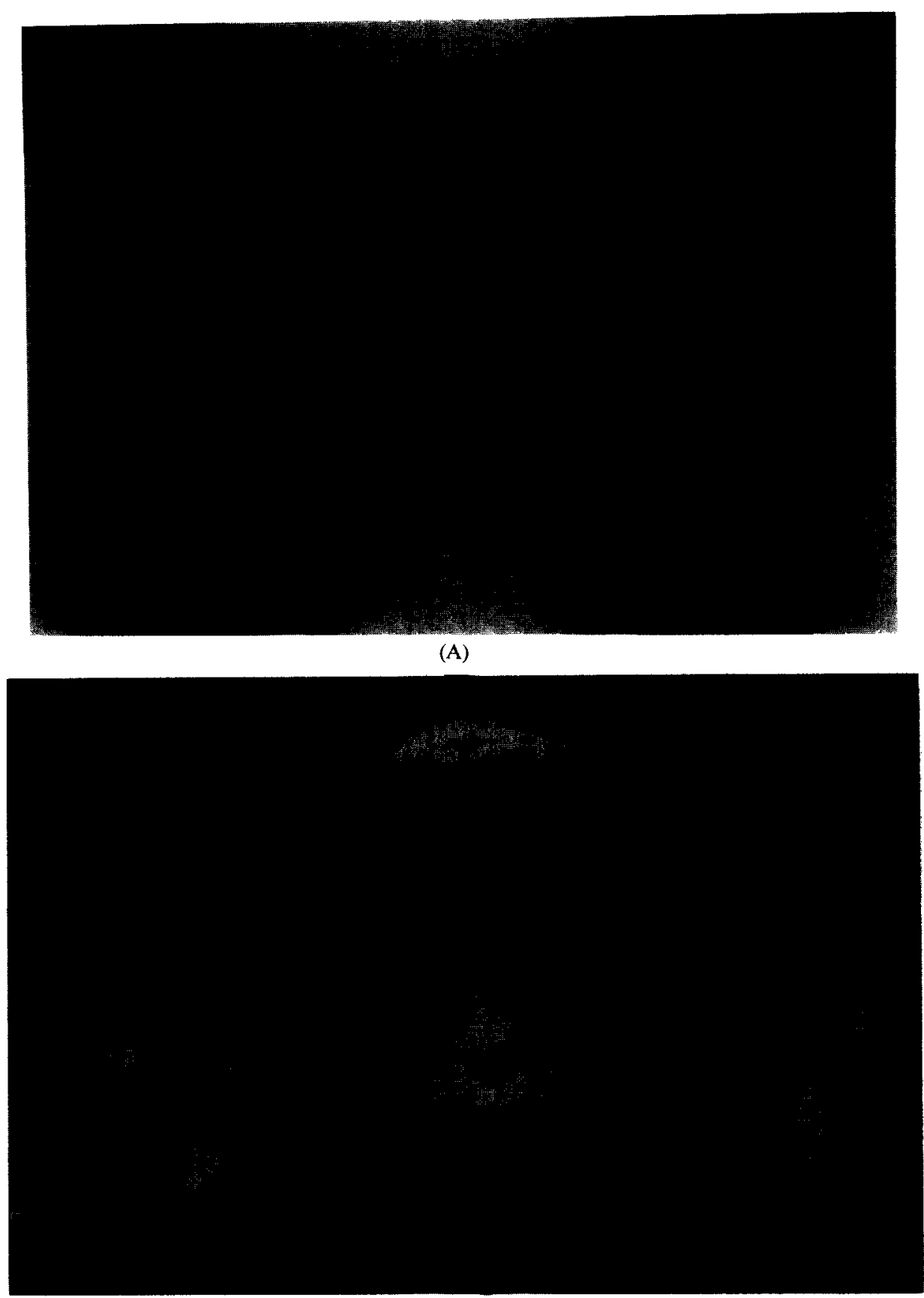

(B)

Fig. 2. Equivocal nodule secondary to prominent costochondral junction. (A) PA chest radiograph shows questionable nodule at left first costochondral junction (arrowhead). (B) CT image demonstrates prominent calcified costochondral cartilage (arrowhead).

drawback of chest fluoroscopy is that it is not nearly as definitive as CT. This is becoming even more of a problem because radiology residency programs do not encourage the development of chest fluoroscopic skills as much as they did in the past.

We feel that the approach to the equivocal pulmonary nodule should rely on the relative advantages of chest fluoroscopy and CT. Chest fluoroscopy can be a fast and inexpensive screening tool for lesions that are not likely to be real nodules. From this study, that would apply to equivocal nodules projecting over two ribs or over the first or second costochondral junction. For all other equivocal nodules (and for patients in whom screening chest fluoroscopy demonstrates an underlying parenchymal abnormality), we recommend CT to distinguish true nodules from scars, airspace disease, normal 


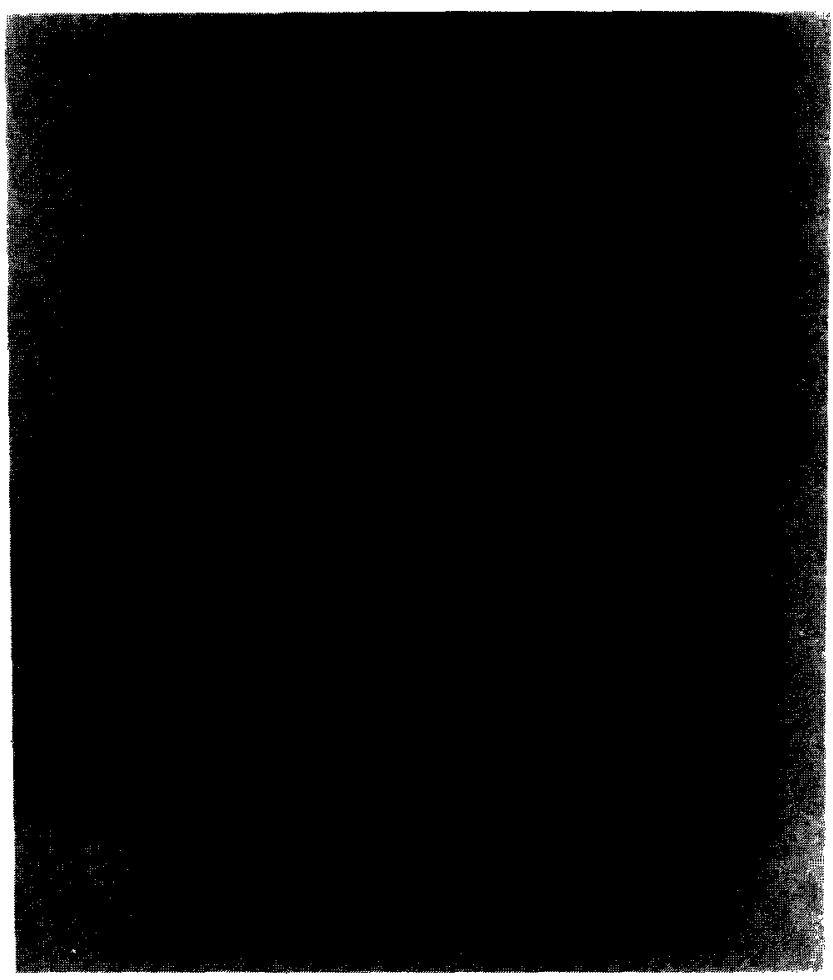

(A)

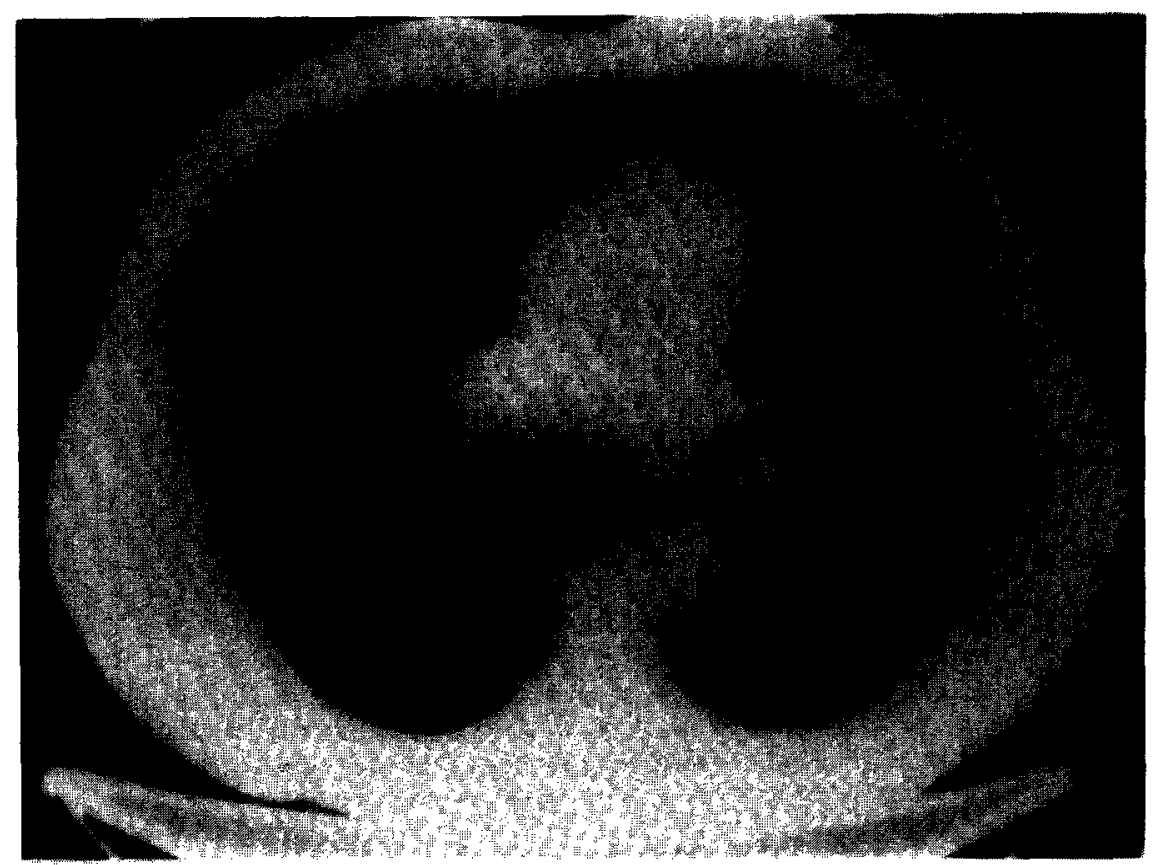

(B)

Fig. 3. Equivocal nodule referred to CT despite radiologic misdiagnosis. (A) PA chest radiograph, officially interpreted as normal, demonstrates a small nodule over the right posterior eighth rib (arrowhead). (B) CT confirms a parenchymal nodule (arrowhead).

variants, or no disease. In patients with true nodules, CT densitometry can be performed, in some cases establishing the presence of diffuse calcification of a nodule.

CT evaluation of equivocal pulmonary nodules is not without problems. The CT demonstration of a small noncalcified nodule does not establish its etiology. Furthermore, some patients will prove 
to have multiple clinically-unsuspected nodules. From our previous CT study of multiple pulmonary nodules, we feel that clinical correlation is very important in patients with one or more noncalcified pulmonary nodules [7]. Closer interval follow-up CT and nodule biopsy are diagnostic alternatives whose appropriateness in a given patient is best decided by the referring physician.

\section{SUMMAR Y}

In the setting of an equivocal pulmonary nodule demonstrated by conventional chest radiographs, CT accurately distinguishes among real nodules, scars, atelectasis or infiltrate, normal structural variants, and no disease. Chest fluoroscopy, because it is fast and inexpensive, is an attractive alternative diagnostic tool for nodules that are unlikely to be real. From our experience this would apply to equivocal nodules projecting over two ribs or over the first or second costochondral junction. For all other equivocal nodules, we suggest CT.

\section{REFERENCES}

1. S. S. Siegelman, E. A. Zerhouni, F. P. Leo, N. F. Khouri and F. P. Stitik, CT of the solitary pulmonary nodule, $A m$. J. Roentg. 135, 1-13 (1980).

2. G. M. Glazer. M. B. Orringer, B. H. Gross and L. E. Quint, The mediastinum in non-small cell lung cancer: CT-surgical correlation, Am. J. Roentg. 142, 1101-1105 (1984).

3. E. G. Schaner, A. E. Chang, J. L. Doppman, D. M. Conkle, M. W. Flye and S. A. Rosenberg, Comparison of computed and conventional whole lung tomography in detecting pulmonary nodules: a prospective radiologic-pathologic study, $\mathrm{Am}$. J. Roentg. 131, 51-54 (1978).

4. J. R. Muhm, L. R. Brown, J. K. Crowe, P. F. Sheedy II, R. R. Hattery and D. H. Stephens, Comparison of whole lung tomography and computed tomography for detecting pulmonary nodules, Am. J. Roentg. 131, 981-984 (1978).

5. R. A. Mintzer, S. R. Malave, H. L. Nieman, L. L. Michaelis, R. M. Vanecko and J. H. Sanders, Computed vs conventional tomography in evaluation of primary and secondary pulmonary neoplasms, Radiology 132, 653-659 (1979).

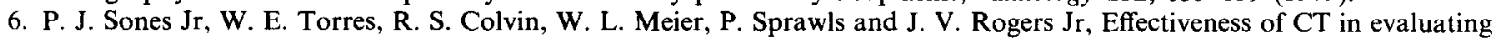
intrathoracic masses, Am. J. Roentg. 139, 469-475 (1982).

7. B. H. Gross, G. M. Glazer and F. L. Bookstein, Multiple pulmonary nodules detected by computed tomography: diagnostic implications, J. Comput. assist. Tomogr. 9, 880-885 (1985).

About the Author-Murray A. Howe received his M.D. from the University of Michigan, where he is currently continuing his training in the radiology residency program.

\footnotetext{
About the Author-Barry II. Gross received his M.D. from the University of Michigan. Following radiulogy residency at the University of Cincinnati, he was a fellow in body computed tomography and ultrasonography at the University of California, San Francisco. He is currently Associate Professor of Radiology, Director, Division of Chest Radiology, and Associate Director, Division of Body Computed Tomography at the University of Michigan.
} 